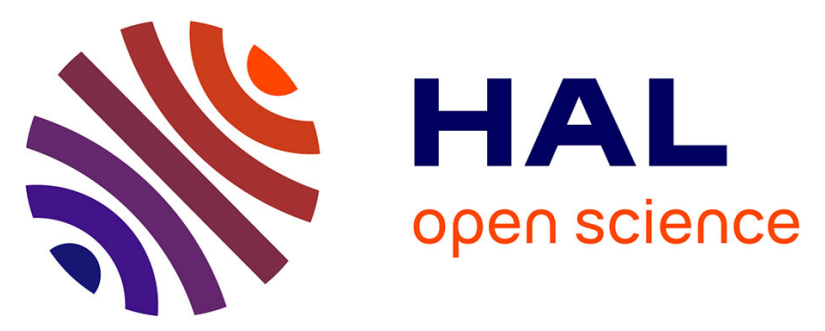

\title{
Contributions de l'ergonomie à la recherche et à l'intervention dans le champ du vieillissement au travail : bref historique.
}

\author{
Catherine Delgoulet, Patrick Le May
}

\section{- To cite this version:}

Catherine Delgoulet, Patrick Le May. Contributions de l'ergonomie à la recherche et à l'intervention dans le champ du vieillissement au travail: bref historique.. Journal de Droit de la Santé et de l'Assurance Maladie, 2018. hal-01770479

HAL Id: hal-01770479

https://hal-univ-paris.archives-ouvertes.fr/hal-01770479

Submitted on 19 Apr 2018

HAL is a multi-disciplinary open access archive for the deposit and dissemination of scientific research documents, whether they are published or not. The documents may come from teaching and research institutions in France or abroad, or from public or private research centers.
L'archive ouverte pluridisciplinaire $\mathbf{H A L}$, est destinée au dépôt et à la diffusion de documents scientifiques de niveau recherche, publiés ou non, émanant des établissements d'enseignement et de recherche français ou étrangers, des laboratoires publics ou privés.

$$
\text { Copyright }
$$


Je suis chargée des politiques sociales et du dialogue social chez Humanis. Mon champ recouvre trois services. Le premier travaille sur la RSE, la mission diversité handicap, les problématiques intergénérationnelles et la qualité de vie au travail. Le second est le service de santé au travail. Le dernier porte sur les relations sociales et le juridique : Humanis étant issu de la fusion de trois groupes de protection sociale, nous avons négocié 60 accords en trois ans et demi, dont un texte instituant un horaire variable flexible, un accord gestion prévisionnelle des emplois et des compétences, un contrat de génération et un accord diversité. Nous tentons à présent de passer un cap supplémentaire au travers de notre premier accord qualité de vie au travail, qui s'inscrit dans un accord de branche récemment signé. II comporte trois volets : l'environnement de travail, l'équilibre entre vie personnelle et professionnelle et la santé au travail. Dans ce dernier cadre, il prend en compte la situation des aidants.

Humanis compte à peu près 6000 personnes. Sa pyramide des âges est plutôt équilibrée, avec 29 \% de 50-64 ans, contre $34 \%$ pour la moyenne française. L'âge moyen est de 42,8 ans.

Nous nous inscrivons dans une logique de gestion prévisionnelle des effectifs, d'adaptation des compétences tout au long de la vie professionnelle, de diversité des âges et transmission intergénérationnelle. Nous avons choisi de faciliter la création de communautés en fonction des goûts et intérêts. Nous nous axons sur la prévention des risques psychosociaux, des troubles musculo-squelettiques et nous nous adaptons aux besoins. Enfin, le dernier enjeu consiste à faciliter la transition vers la retraite.

La pyramide des âges d'Humanis nous a aidés : les départs naturels nous permettent d'éviter un plan de réduction des effectifs. Nous sécurisons le fait que les salariés partent à la retraite dès qu'ils peuvent liquider une retraite à temps plein.

Isabelle Rey

\section{Catherine Delgoulet}

Ergonome, Maître de conférences - HDR à l'Université Paris Descartes, membre du Laboratoire Adaptations Travail Individu, partenaire du GIS-CREAPT

Patrick Le May

Médecin du travail, Centre inter-entreprise et artisanat (Cimiat)

\section{Contributions de l'ergonomie à la recherche et à l'intervention dans le champ du vieillissement au travail : bref historique.}

L'ergonomie est une discipline relativement jeune qui s'est développée au sortir de la seconde guerre mondiale (Laville, 2004). Elle a pour ambition générale de « comprendre le travail pour le transformer » (Guérin et al., 1991) et ainsi d'améliorer les conditions de travail des femmes et des hommes dans un double objectif de santé des populations au travail et de performance des systèmes sociotechniques.

Les enjeux liés au vieillissement au travail ne datent pas d'aujourd'hui et les ergonomes œuvrent dans ce domaine depuis de nombreuses années. Parce que le vieillissement est un processus vécu individuellement et en même temps très dépendant du contexte, ils s'y emploient en considérant trois facettes de la relation âge-travail, sur lesquelles nous reviendrons ici : le vieillissement « par», « par rapport » et « dans » le travail (Volkoff \& Gaudart, 2006).

Cet article propose une approche historique des contributions de l'ergonomie au champ, en replaçant dans le temps les questions économiques et sociales auxquelles les chercheurs et praticiens de l'ergonomie se sont confrontés et la manière dont ils y ont répondu. Trois grandes périodes sont ici identifiées, elles relèvent chacune d'une déclinaison singulière des relations vieillissement au travail depuis les années 60 en France.

\section{Un vieillissement différentiel indexé aux conditions de travail (1960-1980)}

Dès les années 60 et jusqu'à la fin des années 80, une préoccupation majeure a été celle de l'exclusion des salariés âgés du monde du travail. Alors que la seconde guerre mondiale avait eu pour conséquence de mettre ou remettre au travail de nombreuses personnes, dont les plus âgées (Teiger, 1995), la période de trente ans qui suit se caractérise par leur amoindrissement relatif et progressif dans les milieux de travail, à mesure que les baby-boomers, nombreux, et jeunes à l'époque, sont entrés dans la vie active 


\section{DOSSIER THÉMATIQUE}

; ce phénomène étant amplifié par la crise économique de 1975 et les tensions sur le marché de l'emploi qui ne se sont guère taries depuis.

Divers travaux scientifiques ont contribué à la compréhension de ce phénomène à première vue paradoxale : alors que la population générale était en train de vieillir, les plus âgés étaient de plus en plus exclus du travail. Des arguments relatifs à la baisse de productivité, liée à l'affaiblissement des capacités physiques et mentales, ont été avancés, sans que les travaux scientifiques soient en mesure de les confirmer. D'autres ont pointé le rôle des stéréotypes sociaux sur l'âge, qui n'inciteraient ni les employeurs, ni les salariés, à (se) maintenir au travail ou recruter au-delà de 50 ans.

Dans cette même période, les ergonomes ont quant à eux investigué le rôle du travail, de ses conditions et exigences sur ce phénomène d'exclusion lié à l'âge (Laville, Teiger \& Wisner, 1975). Par divers travaux empiriques, ils ont souligné que l'évolution des capacités physiques et cognitives ne pouvait expliquer à elle seule le phénomène observé. Ils ont par ailleurs montré en quoi et comment certaines conditions de travail usent prématurément les salariés et les amènent à quitter le métier occupé, voire le monde du travail.

Trois contraintes ont ainsi été identifiées comme particulièrement délétères au fil de l'âge. II s'agit des horaires décalés (travail posté ou en horaires atypiques, nuits fixes), des contraintes posturo-articulaires (position prolongée debout ou à genoux, piétinement, maintien des bras en l'air, position fixe de la tête ou du cou, etc.) et des efforts physiques intenses (port de charges lourdes), ou enfin de la pression temporelle (travail en urgence ou avec des butées temporelles rigides, cadence fixée par la machine, etc.). Leur cumul dans le travail, qui n'est pas rare, aggrave leur répercussion sur le processus de vieillissement et use prématurément les salariés qui y sont exposés. Ces travaux ont ainsi mis au jour le rôle majeur de ces conditions de travail dans la différenciation du processus de vieillissement d'une personne à une autre (Teiger et al., 1981 ; Teiger, 1989).

En s'intéressant ainsi au vieillissement « par » le travail, les ergonomes se sont attachés à définir les conditions d'une (re) conception d'un travail moins délétère pour la santé à tout âge en jouant notamment sur son organisation, son contenu ou ses conditions physiques et techniques. Leurs investigations ont par ailleurs démontré que les salariés vieillissants ne subissent pas de manière passive le travail, bien au contraire. Elles ont permis d'amorcer un virage représentationnel, en mettant l'accent sur les stratégies opératoires singulières de préservation de la santé et de recherche d'efficacité/qualité au travail (anticiper, réordonner, prioriser, différer, vérifier, coopérer, etc.), susceptibles de faire de l'avancée en âge un atout, à condition que celle-ci soit l'occasion pour chacun(e), dans son quotidien, de se construire une expérience professionnelle ressource. Toutefois, le contexte législatif français de l'époque permettant, voire favorisant, des départs précoces en (pré)retraite, incitait davantage les employeurs à se défaire des populations les plus âgées qu'à transformer leurs systèmes de production ou de service, et les salariés à quitter le monde du travail dès qu'ils en avaient la possibilité compte tenu des pénibilités auxquelles ils étaient confrontés.

\section{Des changements dans le travail qui bousculent les salariés vieillissants (1990-2000)}

Les dix dernières années du XXe siècle se caractérisent par des bouleversements majeurs du travail. Les entreprises initient des changements techniques, organisationnels ou matériels substantiels et se posent de nombreuses questions au sujet des réelles possibilités des salariés plus âgés à suivre le mouvement. Certaines situations sont identifiées comme "critiques » de ce point de vue. Elles doivent être prises au sérieux et nécessitent d'être anticipées pour éviter que d'un statut de salarié(e) expérimenté(e) ou expert d'un domaine, certain(e)s passent au statut de novices, voire de disqualifié(e)s. De nombreux exemples montrent qu'un changement peut venir bouleverser la maîtrise de la situation de travail et des tâches à réaliser, et faire basculer quiconque dans le camp des « trop vieux pour travailler ». Dans ce cadre, c'est notamment l'expérience professionnelle qui fait débat : en période de transformation, peut-elle être une ressource pour l'entreprise et les salarié(e)s ou est-elle un frein?

Au-delà du vieillissement «par » le travail, l'angle de vue vise alors à traiter du vieillissement « par rapport » au travail. Qu'est-ce qui dans le travail fait que, parfois en quelques jours, un salarié passe de référent d'un service ou d'un atelier à trop vieux, usé, dépassé ? Quelles sont ces situations critiques du point de vue de l'âge ? En quoi et comment ces situations tendent-elles à exclure les plus âgés ?

Les travaux en ergonomie vont ainsi contribuer à identifier trois types de situations critiques : l'introduction de nouveaux dispositifs techniques (informatisation, automatisation, etc. ; Marquié, Thon \& Baracat, 1994) ; le déploiement de formations, notamment lorsqu'elles visent exclusivement l'adaptation à la nouvelle situation de travail (Paumès \& Marquié, 1995); et enfin, l'implantation de nouveaux modes managériaux (polyvalence, lean management, etc. ; Gaudart \& Pondaven, 1998). Ces mêmes travaux ont également souligné, avec d'autres, le phénomène d'intensification du travail auquel ces changements ont participé, venant ainsi accroître les contraintes temporelles déjà identifiées comme sélectives sur l'âge.

II s'est alors agi de comprendre à quelles difficultés les plus âgés se confrontent réellement dans les périodes de changements et de définir comment créer les conditions pour ne pas laisser à la porte, de nombreuses évolutions du travail, les salariés vieillissants (Doppler, 1995). En soulignant le rôle de l'expérience et la manière dont elle se développe au fil de l'âge (Gaudart \& Weill-Fassina, 1999), les travaux d'ergonomes ont notamment pu déconstruire l'argument de la « résistance au changement » ou de la «moindre adaptabilité », souvent avancée pour justifier de la mise à 


\section{DOSSIER THÉMATIQUE}

l'écart des plus âgés (Volkoff, Molinié \& Jolivet, 2000). Ils ont montré que ces situations avaient en revanche pour point commun de bousculer l'expérience jusque-là acquise sans fournir, dans bien des cas, les possibilités aux personnes de la redéployer dans des stratégies opératoires qui permettent de tenir conjointement les enjeux de santé et de performance individuelle ou collective (Gaudart, 2000 ; Delgoulet, 2001).

\section{Vieillir au travail .... (2000 -)}

Les années 2000 constituent un tournant dans la manière d'aborder les questions relatives au vieillissement au travail. D'une part les nombreux baby-boomers atteignent ou dépassent la cinquantaine. D'autre part les politiques européennes incitatives autour du "vieillissement actif ", les politiques françaises traduites dans les lois successives sur l'allongement de la vie professionnelle et la disparition progressive des dispositifs de départ anticipé à la retraite, vont rattraper les entreprises et les salariés dans leur projet commun de séparation précoce.

Les questions relatives au vieillissement «par » le travail perdurent, en témoigne le maintien à des niveaux élevés de nombreuses contraintes, posturales ou de cadence (Gollac, Volkoff \&Wolff, 2014). Les questions relatives au vieillissement "par rapport » au travail se voient parfois renforcées, compte tenu du rythme soutenu des changements impulsés par les entreprises, sans véritable stratégie d'accompagnement des plus âgés (Gaudart, 2006). Toutefois, à côté de ces questions, d'autres relatives au maintien des plus âgés au travail et donc au vieillissement « dans » le travail prennent tout leur sens (Molinié, Gaudart \& Pueyo, 2012).

Cette nouvelle configuration renforce l'enjeu de mise au jour de stratégies opératoires « efficientes » qui permettent tout à la fois de construire ou de préserver sa santé et de tenir les objectifs de performance au travail. Dans la lignée des premiers travaux d'ergonomes, les analyses menées récemment montrent que ces stratégies peuvent être individuelles (anticiper les dérives d'un système de production pour se prémunir des interventions d'urgence plus fatigantes à court terme et plus délétères pour la santé à long terme, Pueyo, 2000 ; éviter la douleur ou chercher à la réduire par un usage spécifique de son corps au travail, Chassaing, 2004 ; décaler dans le temps la réalisation certaines tâches pour fiabiliser la qualité du service rendu et se soustraire aux pénibilités du travail de nuit, Toupin, 2012) ; elles peuvent être collectives (réélaborer des règles de travail au sein des collectifs pour faciliter la coopération au bénéfice du service rendu et d'une exposition partagée aux contraintes du travail au contact d'un public, Caroly \& Weill-Fassina, 2004 ; répartir de manière informelle des tâches en fonction des capacités et de l'expertise de chacun pour la sécurité de tous, Zara-Meylan, 2006); ou encore organisationnelles (gérer des parcours de travail au regard des exigences des postes et de l'évolution des capacités de chacun au fil de l'âge, Gonon, 2003 ; penser de nouvelles fonctions en entreprise pour accroître les marges de reclassement, en étant au plus près des compétences que les salariés ont pu développer avec l'expérience, Caron et al., 2012). Ces travaux pointent l'intelligence et la créativité que chacun(e) mobilise dans la pratique professionnelle au quotidien. Ils soulignent la diversité et la variabilité des situations et des personnes, deux notions chères à l'ergonomie depuis son origine.

Il s'agit alors de mettre en visibilité l'efficience de ces stratégies opératoires, de concourir à leur reconnaissance et de les promouvoir tout au long de la vie. Alors que la tendance de fond dans les entreprises est à la standardisation, des process jusqu'aux modes opératoires de réalisation des tâches, traiter du vieillissement « dans » le travail revient donc à (re)concevoir le travail pour qu'il soit un opérateur de santé et de développement au même titre qu'un vecteur de performance, dans un souci de prise en compte de la diversité et de la variabilité des personnes et des situations professionnelles.

\section{Comprendre les relations vieillissement $x$ travail dans ses trois dimensions et agir}

Considérer les trois facettes du vieillissement au travail (par, par rapport et dans) fournit aux ergonomes des outils pour agir, en pluridisciplinarité (services de médecine du travail, de prévention et gestion des risques, $\mathrm{RH}$, ingénierie de production ou de conception), à divers niveaux dans les entreprises.

Les actions peuvent ainsi concerner la mise en place et la création de démarches d'anticipation, d'accompagnement et d'évaluation aux niveaux individuel, collectif ou organisationnel. A titre d'exemple citons ici certaines d'entre elles (pour le détail voir, Caron et al., 2012) :

Les dispositifs d'évaluation et de suivi des conditions de travail et de la santé des salariés permettent à la fois de prendre une photo détaillée des principales caractéristiques du travail et de leurs effets sur la santé, et dans une utilisation longitudinale de prendre la mesure de leurs évolutions conjointes. Ils sont des outils de diagnostic mais aussi d'alerte si l'on prend soin d'analyser les signes avant-coureurs de difficultés de santé qu'ils mettent au jour (cf. l'observatoire EVREST, Molinié \& Leroyer, 2011) ; ils participent à l'amélioration globale des conditions de travail dans les entreprises ;

La gestion collective des situations de travail concerne des démarches qui sont initialement élaborées dans le cadre d'une action spécifique auprès de personnes vieillissantes confrontées à un problème individuel et qui aboutissent à une disposition bénéfique pour un ensemble de salariés susceptibles d'être intéressés ou concernés. On trouve par exemple dans ces actions celles qui visent à ré-internaliser des postes jusqu'ici pris en charge par des contrats de sous-traitance; une mesure qui peut, à conditions que l'on ait au préalable mené une évaluation précise des exigences associées à ces postes, élargir les opportunités de reclassement de personnes en difficulté au 


\section{DOSSIER THÉMATIQUE}

poste actuellement occupé ;

une gestion collective des parcours professionnels, qui permet aux salariés de quitter de manière temporaire ou progressive certaines conditions de travail reconnues sélectives sur l'âge, via par exemple la mise en place de missions de travail en journée pour des salariés travaillant en nuits fixes ; par l'implication dans des tâches de tutorat qui sont des occasions de se soustraire aux contraintes de production et de valoriser son expérience ; par la création de nouvelles fonctions dans une visée stratégique d'évolution d'une entreprise et d'offres de nouvelles opportunités de reclassement; autant de mesures profitables aux entreprises qui les mettent en place et aux salariés qui en bénéficient;

Une prise en charge des individus, dans le cadre par exemple de commissions pour le maintien en emploi ou de démarches d'aménagement spécifique de leur poste de travail, du contenu de leur travail, etc. (Quériaud, Dugué \& Petit, 2015).

Travailler à partir des trois facettes du vieillissement au travail et non dans une perspective de "gestion des âges ", tant dans la compréhension des problèmes que dans l'action, favorise une approche décloisonnée de ces questions (Delgoulet et al., 2014). Cette approche évite d'isoler :

les domaines d'action : le vieillissement au travail concerne à la fois les questions de formation, de conditions de travail, de recrutement, etc.

les cibles : ne pas se préoccuper que des « seniors » mais aussi de ceux qui vont vieillir au travail, dans des conditions plus ou moins favorables ;

les focales d'intervention : en naviguant entre la population cible générale (les salariés d'une entreprise) et les individus dans leur singularité ;

les fonctions des outils, qui sont à la fois des outils de veille, de diagnostic et de suivi pour alerter et guider le choix des actions à retenir, leur mise en œuvre effective et leur évaluation ;

les temps de l'action, puisqu'il convient de pérenniser l'approche et non de mener des actions coup de poing étant donné que l'infléchissement des courbes démographiques prend du temps, le développement des compétences aussi, ce qui suppose que ce soit dans la durée et non dans l'urgence que se traitent ces questions.

Ces cinq formes de « décloisonnement » (le domaine d'action, la cible, la focale d'intervention, la fonction des outils, la temporalité des actions), insistent sur le caractère intégré des questions de maintien en emploi des salariés les plus âgés. Les catégories d'âge ou autres, parfois utiles pour donner des orientations de l'action publique et cibler des priorités, pourraient en revanche s'avérer restrictives pour agir en situation et évaluer l'action. En isolant notamment la catégorie des « seniors » on court le risque de la stigmatisation et de la marginalisation. Ce principe de décloisonnement, porté par l'ergonomie dans son approche du vieillissement au travail, permet au contraire de jeter les ponts entre ces catégories et d'estomper leur coupure artificielle. II invite à penser le travail et la formation dans la durée des parcours de salariés (Delgoulet \& Vidal-Gomel, 2013), et contribue à la conception d'outils, d'organisations et situations de travail « capacitants » (Falzon, 2013), c'est-à-dire favorables au développement des femmes et des hommes tout au long de leur vie professionnelle.

Catherine Delgoulet

\section{Bibliographie}

- Caroly, S., \& Weill-Fassina, A. (2004). Évolutions des régulations de situations critiques au cours de la vie professionnelle dans les relations de service. Le Travail Humain, 67(4), 305-332.

- Caron, L., Caser, F., Delgoulet, C., Effantin, E., Jolivet, A., Théry, L., \& Volkoff, S. (2012). Les conditions de travail dans les accords et plans d'action « seniors ». Rapport de recherche du CEE, $n^{\circ} 79$, juillet. http://www.ceerecherche.fr/fr/c_pub4.htm

- Chassaing, K. (2004). Vers une compréhension de la construction des gestuelles avec l'expérience : le cas des « tôliers » d'une entreprise automobile. Pistes, 6(1). http://pistes.revues.org/3280

- Delgoulet, C. (2001). La construction des liens entre situations de travail et situations d'apprentissage dans la formation professionnelle. PISTES. 3(2). http://www. pistes.uqam.ca

- Delgoulet, C., \& Vidal-Gomel, C. (2013). Le développement des compétences : une condition pour la construction de la santé et de la performance au travail. In, P., Falzon (Ed), Ergonomie constructive (pp 19-32), Paris : PUF.

- Delgoulet, C., Volkoff, S., Caron, L., Caser, F., Jolivet, A., \& Théry, L. (2014). Conditions de travail et maintien en emploi des seniors : les enjeux d'un décloisonnement des approches et des expérimentations. Quelques enseignements issus de monographies d'entreprises françaises. Relations Industrielles/Industrial Relations, 69(4), 687-708.

- Doppler, F. (1995). Évolution de la population et transformation ou conception des situations de travail. In, J.C. Marquié, D. Paumès, et S. Volkoff (Eds.), Le travail au fil de l'âge (pp. 411-423), Toulouse : Octarès.

- Falzon, P. (Coord.) (2013). Ergonomie constructive. Paris :PUF.

- Gaudart, C. (2000). Conditions for maintaining ageing operators at work-a case study conducted at an automobile manufacturing plant. Applied Ergonomics, 31(5), 435-462.

- Gaudart, C. (2006). Les âges au travail. In, Théry Laurence (dir.). Le travail intenable : résister collectivement à l'intensification du travail (pp. 119-137), Paris : La Découverte. 


\section{DOSSIER THÉMATIQUE}

- Gaudart, C., \& Podaven, S. (1998). Polyvalence, vieillissement et expérience dans deux métiers de la sidérurgie, Actes du XXXIIIle Congrès de la SELF» Temps et Travail, Paris.

- Gaudart, C., \& Weill-Fassina, A. (1999). L'évolution des compétences au cours de la vie professionnelle : une approche ergonomique. Formation emploi, 67, 47-62.

- Gollac, M., Volkoff, S., \& Wolff, L. (2014). Les conditions de travail. Paris : La Découverte, Repères.

- Gonon, O. (2003). Des régulations en lien avec l'âge, la santé et les caractéristiques du travail : le cas des infirmières d'un centre hospitalier français. Pistes, 5(1). http://pistes.revues.org/3336

- Guérin, F., Laville, A., Daniellou, F., Duraffourg, J., \& Kerguelen, A. (1991). Comprendre le travail pour le transformer. La pratique de l'ergonomie. Lyon : éditions de l'ANACT.

- Laville, A. (2004). Repères pour une histoire de l'ergonomie francophone. In, P., Falzon (Ed), Ergonomie constructive (pp 37-50), Paris : PUF.

- Laville, A., Teiger, C., \& Wisner, A. (Coord) (1975). Age et contraintes de travail. Jouy en Josas: N.E.B.

- Marquié, J.-C., Thon, B., \& Baracat, B. (1994). Age influence on attitudes of office workers faced with new computerized technologies. Applied Ergonomics, 25(3), 130-142.

- Molinié, A.-F., \& Leroyer, A. (2011). Suivre les évolutions du travail et de la santé : EVREST, un dispositif commun pour des usages diversifiés, Perspectives interdisciplinaires sur le travail et la santé, 13(2). http://pistes.revues. org/1852

- Molinié, A.F., Gaudart, C., \& Pueyo, V. (Coord.) (2012). La vie professionnelle : âge, expérience et santé à l'épreuve des conditions de travail. Toulouse : Octarès.

- Paumès, D., \& Marquié, J.-C. (1995). Travailleurs vieillissants, apprentissage et formation professionnelle. In J.-C. Marquié, D. Paumès, \& S. Volkoff (Eds.), Le travail au fil de l'âge (pp. 391-410). Toulouse : Octarès.

- Pueyo, V. (2000). La traque des dérives : expérience et maîtrise du temps, les atouts des anciens dans une tâche d'autocontrôle. Travail et emploi, 84, 63-73.

- Quériaud, C., Dugué, B., \& Petit, J. (2015). Les enjeux organisationnels de la reprise du travail de salariés après une longue absence, Perspectives interdisciplinaires sur le travail et la santé, 17(1). http://pistes.revues.org/4469.

- Teiger, C. (1989). Le vieillissement différentiel dans et par le travail, un vieux problème dans un contexte récent. Le Travail humain, 52(1), 21-56.

- Teiger, C. (1995). Penser les relations âge/travail au cours du temps. In J.-C. Marquié, D. Paumès, \& S. Volkoff (Eds.), Le travail au fil de l'âge (pp. 15-72). Toulouse : Octarès.
- Teiger, C., Laville, A., Lortie, M., Binder, E., \& Boutin, J. (1981). Travailleurs de nuit permanents : rythmes circadiens et mortalité. Le Travail Humain, 44, 71-92.

- Toupin, C. (2012). L'expérience du travail de nuit chez les infirmières de pneumologie. In, A.-F Molinié, C. Gaudart \& V. Pueyo (Eds.), La vie professionnelle : âge, expérience et santé à l'épreuve des conditions de travail (pp. 161177). Toulouse : Octarès.

- Volkoff, S., \& Gaudart, C. (2006). Vieillissement et travail. In M. Goldberg (coord.), La place de la santé au travail dans la santé publique. Actualité et Dossier en Santé Publique, 57, 52-54.

- Volkoff, S., Molinié, A.-F., \& Jolivet, A. (2000). Efficaces à tout âge ? Dossiers du CEE, $n^{\circ} 16$.

- Zara-Meylan, V. (2006). Contraintes organisationnelles et gestion des risques en milieu ouvert : l'activité des monteurs installateurs de structures de fête, Perspectives interdisciplinaires sur le travail et la santé, 8(1). http://pistes.revues.org/3087 


\section{DOSSIER THÉMATIQUE}

Globalement, la santé générale de la population s'améliore : les personnes vivent mieux et plus longtemps. De même, les conditions de travail tendent à s'améliorer. Pour autant, le vieillissement est un processus inévitable. II est soumis à des déterminants génétiques et environnementaux, notamment les conditions de travail. II se traduit par une « usure » physique, mentale et sensorielle et une prévalence plus importante de maladies chroniques, notamment dégénératives. Le travail peut révéler, accélérer ou amplifier des déficits, par des contraintes physiques ou temporelles.

La prévention primaire est primordiale. Je n'insiste pas sur la prévention traditionnelle : nutrition, activité physique, hygiène de vie, prévention et dépistage, bilans de santé... Les visites de médecine du travail participent à cette prévention. Fondée en 1946, la médecine du travail a reposé depuis des années sur la visite d'embauche et la visite annuelle pour tous. Ces visites se sont peu à peu espacées pour atteindre un rythme quinquennal aujourd'hui. La loi prévoit une surveillance individuelle renforcée pour certains risques : Amiante, Plomb, certains agents CMR (cancérogènes, mutagènes, reprotoxiques), certains agents biologiques, les rayonnements ionisants, le risque hyperbare, le risque de chute de hauteur lors des opérations de montage et de démontage des échafaudages, certains postes nécessitant un examen d'aptitude spécifique. Des visites individuelles adaptées concernent les travailleurs handicapés, les travailleurs en invalidité, les travailleurs de nuit et deux critères soumis à l'appréciation du médecin du travail : les conditions de travail et l'âge.

Sur le plan collectif, la prévention passe par un allégement du travail physique, l'optimisation de l'organisation du travail, l'adaptation de l'environnement de travail et la formation. L'objectif majeur est le maintien dans l'emploi. Pour ce faire, on peut procéder à des aménagements et des contreindications : limitation de la charge physique, télétravail, temps partiel thérapeutique. Encore faut-il que la limitation ne vide pas le poste de son contenu. Un retrait provisoire du poste s'impose parfois : le médecin du travail n'a pas vocation à faire un arrêt de travail, mais oriente vers les structures de soins pour cela. Une mise en invalidité ou une Reconnaissance de la Qualité de Travailleur Handicapé peut également s'avérer nécessaire. Enfin, en cas d'inaptitude médicale définitive, le reclassement dans l'entreprise s'impose, sauf en cas de risque psychosocial majeur. L'employeur doit prendre en compte les recommandations du médecin du travail pour reclasser le salarié, mais cela peut être difficile, surtout pour les salariés âgés ou encore dans les TPE. La conclusion est souvent le licenciement.

Patrick Le May 